\title{
Exotic few-body bound states in a lattice
}

\author{
D. Petrosyan and M. Valiente \\ Institute of Electronic Structure and Laser, Foundation for Research and Technology-Hellas, 71110 Heraklion, Crete, Greece
}

\begin{abstract}
Strongly-interacting ultra-cold atoms in tight-binding optical lattice potentials provide an ideal platform to realize the fundamental Hubbard model. Here, after outlining the elementary single particle solution, we review and expand our recent work on complete characterization of the bound and scattering states of two and three bosonic atoms in a one-dimensional optical lattice. In the case of two atoms, there is a family of interaction-bound "dimer" states of co-localized particles that exists invariantly for either attractive or repulsive on-site interaction, with the energy below or above the two-particle scattering continuum, respectively. Adding then the third particle - "monomer" we find that, apart from the simple strongly-bound "trimer" corresponding to all three particles occupying the same lattice site, there are two peculiar families of weakly-bound trimers with energies below and above the monomer-dimer scattering continuum, the corresponding binding mechanism being an effective particle exchange interaction.
\end{abstract}

\section{INTRODUCTION}

Among the tight-binding lattice models of condensed matter physics[1, 2], the Hubbard model [3] plays a fundamental role. It describes particle tunneling between adjacent lattice sites as well as short range (contact) interaction between the particles on the same lattice site. Despite apparent simplicity, this model is very rich in significance and implications for the many body physics on a lattice [4]. This is perhaps most profoundly manifested with numerous important experimental and theoretical achievements with cold neutral atoms trapped in deep optical lattice potentials [5 7], wherein the Hubbard model is being realized with unprecedented accuracy.

A remarkable Hubbard model phenomenon is the existence of stable repulsively-bound pairs of atoms in an optical lattice, as was experimentally demonstrated in Ref. [8]. This seminal achievement has led to several theoretical studies of the properties of interaction-bound atom pairs - "dimers" - in periodic potentials 9 -19].

In a one-dimensional (1D) tight-binding lattice, two bosons can form a bound dimer 8 13] for any finite strength of the on-site interaction, be it an attraction or a repulsion. Next level of complexity corresponds to three bosons, which obviously can form a strongly-bound "trimer" with all three particles occupying the same lattice site. For large enough on-site interaction strength, however, there are two more kinds of weakly bound trimers [20]. The corresponding binding mechanism turns out to be an effective particle exchange interaction between the dimer and the third particle - "monomer" - leading to symmetric and antisymmetric trimer states with energies slightly above and below the continuum of scattering states of (asymptotically) free dimer and monomer.

Below, after introducing the Bose-Hubbard model and outlining its elementary single particle solution, we review and expand our recent work [12,20] on the bound and scattering states of two and three bosonic atoms in a 1D optical lattice.

\section{THE MODEL}

Cold bosonic particles in a 1D tight-binding lattice can be accurately described by the (second-quantized) Hubbard Hamiltonian [3 7]

$$
H=-J \sum_{j}\left(\hat{b}_{j}^{\dagger} \hat{b}_{j+1}+\hat{b}_{j+1}^{\dagger} \hat{b}_{j}\right)+\frac{U}{2} \sum_{j} \hat{n}_{j}\left(\hat{n}_{j}-1\right)
$$

where $\hat{b}_{j}^{\dagger}\left(\hat{b}_{j}\right)$ is the particles creation (annihilation) operator and $\hat{n}_{j}=\hat{b}_{j}^{\dagger} \hat{b}_{j}$ the number operator at $j$ th lattice site; $J(>0)$ is the inter-site tunneling, or hopping, rate; and $U$ is the on-site interaction, which can be attractive or repulsive.

a. Single particle solution: Denoting by $|j\rangle$ the state with a single particle at the $j$ th lattice site, the Hamiltonian reduces to

$$
H^{(1)}=-J \sum_{j}(|j\rangle\langle j+1|+| j+1\rangle\langle j|) .
$$




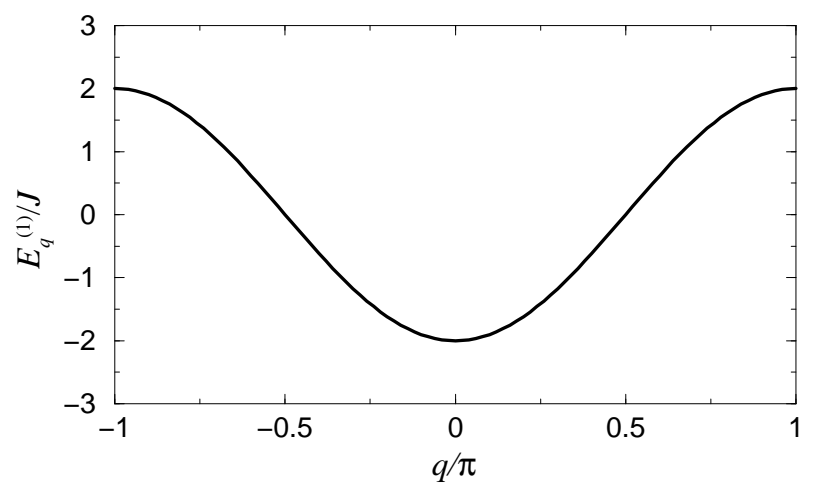

FIG. 1: Single particle Bloch band in a 1D tight-binding lattice.

Expanding the single-particle state vector as $|\psi\rangle=\sum_{j} \psi(j)|j\rangle$, the stationary Schrödinger equation $H^{(1)}|\psi\rangle=$ $E^{(1)}|\psi\rangle$ leads to the difference equation

$$
-J[\psi(j+1)+\psi(j-1)]=E^{(1)} \psi(j),
$$

which is satisfied by the (discrete) plane wave ansatz $\psi(j)=\psi_{q}(j)=\exp (i q j)$ for the wave function with quasimomentum $q \in \Omega$ restricted to the first Brillouin zone $\Omega \equiv[-\pi, \pi]$, while for the corresponding eigenenergy we obtain $E_{q}^{(1)}=\epsilon(q) \equiv-2 J \cos (q)$. The single-particle energy (Bloch) band, shown in Fig. 1, has, therefore, a width of $4 J$.

Having reviewed the trivial single-particle solution [1], in the following Sections we present complete solutions of the much richer two- and three-body problems in a 1D lattice.

\section{TWO PARTICLES IN A LATTICE}

Although the Bose-Hubbard Hamiltonian (1) corresponds to identical bosons, which is our main concern here, it is nevertheless instructive to tackle the more general problem of two distinguishable particles in a lattice [11], a simple limit of which produces the solution for two indistinguishable bosons.

We thus consider two particles $A$ and $B$ having, in general, different hopping rates $J_{A}$ and $J_{B}$, respectively. The Hamiltonian describing their dynamics is the non-symmetrized (first-quantized) version of the Hubbard Hamiltonian (11) acting on the two particle subspace:

$$
H^{(2)}=-J_{A} \sum_{j_{A}}\left(\left|j_{A}\right\rangle\left\langle j_{A}+1|+| j_{A}+1\right\rangle\left\langle j_{A}\right|\right)-J_{B} \sum_{j_{B}}\left(\left|j_{B}\right\rangle\left\langle j_{B}+1|+| j_{B}+1\right\rangle\left\langle j_{B}\right|\right)+U \sum_{j_{A}=j_{B}}\left|j_{A}, j_{B}\right\rangle\left\langle j_{A}, j_{B}\right|,
$$

where $j_{A}$ and $j_{B}$ denote the positions of particles $A$ and $B$, respectively. The eigenstates of $H^{(2)}$ can be expanded as $|\Psi\rangle=\sum_{j_{A}, j_{B}} \Psi\left(j_{A}, j_{B}\right)\left|j_{A}, j_{B}\right\rangle$, so that the Schrödinger equation $H^{(2)}|\Psi\rangle=E^{(2)}|\Psi\rangle$ leads to the difference equation

$$
-J_{A}\left[\Psi\left(j_{A}+1, j_{B}\right)+\Psi\left(j_{A}-1, j_{B}\right)\right]-J_{B}\left[\Psi\left(j_{A}, j_{B}+1\right)+\Psi\left(j_{A}, j_{B}-1\right)\right]+U \delta_{j_{A}, j_{B}} \Psi\left(j_{A}, j_{B}\right)=E^{(2)} \Psi\left(j_{A}, j_{B}\right) .
$$

In order to solve the problem analytically, we need to transform the two-body difference equation (5) into a "onebody" problem. To that end, we define the center of mass $j_{R}=\frac{1}{2}\left(j_{A}+j_{B}\right)$ and relative $j_{r}=j_{A}-j_{B}$ coordinates and use for the two-particle wave function the separation ansatz [15, 20]

$$
\Psi\left(j_{A}, j_{B}\right)=e^{i K j_{R}} e^{-i \beta_{K} j_{r}} \psi_{K}\left(j_{r}\right),
$$

where

$$
\tan \left(\beta_{K}\right)=\frac{J_{A}-J_{B}}{J_{A}+J_{B}} \tan (K / 2)
$$

with $K \in \Omega$ the center-of-mass quasimomentum. Note that for $J_{A}=J_{B}=J$ the separation ansatz (6) reduces to that for identical particles [12, 14]. The resulting recursion relation now reads

$$
-J_{K}\left[\psi_{K}\left(j_{r}+1\right)+\psi_{K}\left(j_{r}-1\right)\right]+U \delta_{j_{r}, 0} \psi_{K}\left(j_{r}\right)=E_{K}^{(2)} \psi_{K}\left(j_{r}\right),
$$


where $J_{K}=\sqrt{J_{A}^{2}+J_{B}^{2}+2 J_{A} J_{B} \cos (K)}$ is the collective hopping rate [11, 15], which in the case of identical particles, $J_{A}=J_{B}=J$, reduces to the standard [8, 9, 12, 14] expression $J_{K}=2 J \cos (K / 2)$.

Equation (8) admits two kinds of solutions, corresponding to the scattering states of asymptotically free particles and to the two-particle bound, or dimer, states.

\section{A. Scattering states}

Since the interaction between the particles is governed by a short-range - in this case a contact, $U \delta_{j_{r}, 0}-$ potential, its action amounts to a unitary phase shift (see below), while the spectrum of such solutions is given by the sum of the spectra for two free particles $A$ and $B$ with momenta $q_{A}=K / 2+k$ and $q_{B}=K / 2-k$ :

$$
E_{K, k}^{(2)}=E_{q_{A}}^{(1)}+E_{q_{B}}^{(1)}=-2 J_{K} \cos (k),
$$

which spans the interval $E_{K, k}^{(2)} \in\left[-2 J_{K}, 2 J_{K}\right]$. The corresponding symmetric scattering wave functions, for $\sin (k) \neq 0$, are given by

$$
\psi_{K, k}\left(j_{r}\right)=\cos \left(k\left|j_{r}\right|+\delta_{K, k}\right),
$$

which, upon substitution into Eq. (8), yields for the scattering phase shift $\delta_{K, k}$,

$$
\tan \left(\delta_{K, k}\right)=-\frac{U}{2 J_{K} \sin (k)}
$$

For $U \rightarrow 0$ we have non-interacting particles $\psi_{K, k}\left(j_{r}\right)=\cos \left(k\left|j_{r}\right|\right)$, while for $U / J_{K} \rightarrow \pm \infty$ we obtain the fermionized solution $\psi_{K, k}\left(j_{r}\right)=\sin \left(k\left|j_{r}\right|\right)$, whereby the two particles never occupy the same lattice site, $\psi_{K, k}(0)=0$.

When $\sin (k)=0$, the scattering wave functions (10) are no longer valid, and the lattice generalization of the continuum zero-energy solution of the Schrödinger equation apply [10, 15]. At the bottom and the top of the scattering band, $E_{K, \mp}^{(2)}=\mp 2 J_{K}(k=0, \pi)$, the corresponding solutions $\psi_{K, \mp}$ have the form

$$
\begin{aligned}
& \psi_{K,-}\left(j_{r}\right)=1-\frac{\left|j_{r}\right|}{a_{K,-}} \\
& \psi_{K,+}\left(j_{r}\right)=(-1)^{j_{r}}\left(1-\frac{\left|j_{r}\right|}{a_{K,+}}\right),
\end{aligned}
$$

where $a_{K, \mp}$, with $a_{K,-}=-a_{K,+}$, are the scattering lengths, which are calculated by substituting Eqs. (12) into Eq. (8) , resulting in $a_{K,-}=-2 J_{K} / U$ (in units of the lattice constant). Note that, since the scattering lengths are finite for any $U \neq 0$, there exists no "zero-energy" resonance in this model. [27] Therefore, for every value of the total quasimomentum $K$ there is only one bound state, as we shall see below.

To illustrate the foregoing discussion, in Fig. 2 we show the energy spectrum (9) for two identical bosons, $J_{A}=$ $J_{B}=J$, and the corresponding density of states defined via

$$
\rho(E, K)=\frac{L}{2 \pi} \frac{\partial k}{\partial E}=\frac{L}{2 \pi} \frac{1}{\sqrt{[4 J \cos (K / 2)]^{2}-E^{2}}},
$$

with $L$ a quantization length. As seen, the density of scattering states, i.e., the number of states per unit interval of energy, is lowest in the middle of the band, $E \simeq 0$ (and $K \simeq 0$ ), while for a given quasimomentum $K$ of the center of mass motion of the two particles, $\rho(E, K)$ increases rapidly as energy $E=E_{K, k}^{(2)}$ approaches its maximal and minimal values $\pm 2 J_{K}$.

\section{B. Bound states}

The two-body bound states of Hamiltonian (44) are the solutions of Eq. (8) yielding normalizable relative coordinate wave function, $\sum_{j_{r}}\left|\psi_{K}\left(j_{r}\right)\right|^{2}<\infty$, with the corresponding energy below (for $U<0$ ) or above (for $U>0$ ) the scattering continuum at each value of the total quasimomentum $K$. Introducing into Eq. (8) the exponential ansatz $\psi_{K}\left(j_{r}\right) \propto \alpha_{K}^{\left|j_{r}\right|}$ yields the bound state energy $E_{K}^{(2)}=-J_{K}\left(\alpha_{K}+1 / \alpha_{K}\right)$, with $\alpha_{K}$ given by

$$
\alpha_{K}=\mathcal{U}_{K}-\operatorname{sgn}(U) \sqrt{\mathcal{U}_{K}^{2}+1}, \quad \mathcal{U}_{K} \equiv \frac{U}{2 J_{K}} .
$$




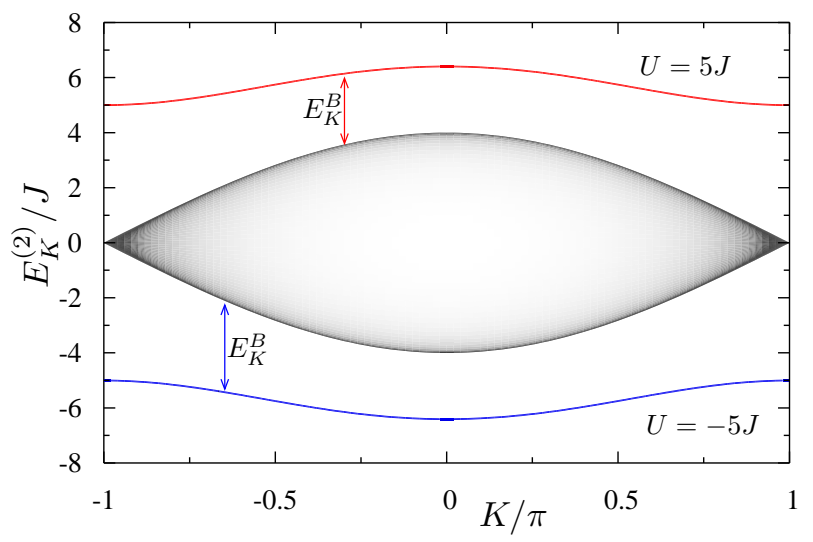

FIG. 2: Energies $E_{K}^{(2)}$ versus the center-of-mass quasimomentum $K$ for a pair of identical bosons in a $1 \mathrm{D}$ lattice. The continuum spectrum corresponds to energies (9) of the scattering states, with the shading proportional to the density of states (13). The (blue) line below and the (red) line above the scattering band are, respectively, the energies (14) of the attractively-bound dimer with $U=-5 J$ and the repulsively-bound dimer with $U=5 J$.

We see that $\alpha_{K}$ is real and also $\left|\alpha_{K}\right|<1$ for all $U \neq 0$. Hence, the relative coordinate wave function $\psi_{K}\left(j_{r}\right)$ is normalizable, and the energy satisfies $\left|E_{K}^{(2)}\right|>2 J_{K}$ for all $K$. Explicitly, the energy and the normalized wave function for the bound dimer are given by

$$
\begin{aligned}
E_{K}^{(2)} & =\operatorname{sgn}(U) \sqrt{U^{2}+4 J_{K}^{2}}, \\
\psi_{K}\left(j_{r}\right) & =\frac{\sqrt{\left|\mathcal{U}_{K}\right|}}{\sqrt[4]{\mathcal{U}_{K}^{2}+1}}\left(\mathcal{U}_{K}-\operatorname{sgn}(U) \sqrt{\mathcal{U}_{K}^{2}+1}\right)^{\left|j_{r}\right|} .
\end{aligned}
$$

In Fig. 2 we show the energies $E_{K}^{(2)}$ for a pair of identical bosons, $J_{A}=J_{B}=J$, interacting via on-site attractive, $U<0$, or repulsive, $U>0$, potential. The binding energies $E_{K}^{B}$ are defined with respect to the edges of the scattering band (91). Note that in the case of repulsive interaction, $U>0\left(\alpha_{K}<0\right)$, the sign of the wave function (15) alternates between the neighboring sites $j_{r}$. Remarkably, when $|K|=\pi$, and thereby $J_{K}=0$, the relative coordinate wave function $\psi_{K}\left(j_{r}\right)$ is completely localized at $j_{r}=0$ for any $U \neq 0$.

Clearly, for a given value of the dimer quasimomentum $K$, the stronger is the on-site interaction $|U|$, the smaller is the extent of the wave function $\psi_{K}\left(j_{r}\right)$, meaning that the constituent particles are stronger co-localized. For $|U| \gg J$, the dimer energy in Eq. (14) can be approximated as

$$
E_{K}^{(2)} \simeq \mathcal{E}^{(2)}-2 J^{(2)} \cos (K),
$$

where the first term $\mathcal{E}^{(2)} \equiv\left[U-2 J^{(2)}\right]$ represents the dimer "internal energy", while the second term $\epsilon^{(2)}(K) \equiv$ $-2 J^{(2)} \cos (K)$ is the kinetic energy of a dimer with quasimomentum $K$ and an effective tunnelling rate $J^{(2)} \equiv-2 J^{2} / U$.

\section{THREE PARTICLES IN A LATTICE}

Building on the solution of the two-body problem, in this section we consider three bosonic atoms is an optical lattice described by Hamiltonian (1).

\section{A. Spectrum of scattering states}

With the expertise gained from the previous section, we can readily deduce that in the case of three particles there are two distinct scattering continua (see Fig. 3 left panel). The first is the three-body scattering continuum of three (asymptotically) free particles, with the energy given by the sum of single-particle bands, $E_{c 3}=\epsilon\left(k_{1}\right)+\epsilon\left(k_{2}\right)+\epsilon(K-$ $\left.k_{1}-k_{2}\right) \equiv \epsilon\left(k_{1}, k_{2}, K-k_{1}-k_{2}\right)$, where each particle quasimomentum $k_{j} \in \Omega$ is in the first Brillouin zone $\Omega \equiv[-\pi, \pi]$ and $K=k_{1}+k_{2}+k_{3}(\bmod 2 \pi)$ is the total quasimomentum. The second is the two-body scattering continuum of a bound pair (dimer) and a free particle (monomer), with energy $E_{c 2}=\operatorname{sgn}(U) \sqrt{U^{2}+[4 J \cos (Q / 2)]^{2}}-2 J \cos (K-Q)$, where the first term is the energy of a dimer, Eq. (14), with quasimomentum $Q$. 


\section{B. Bound states}

We seek the bound states $|\Psi\rangle$ of three bosons in momentum representation,

$$
|\Psi\rangle=\frac{1}{(2 \pi)^{3 / 2}} \iiint_{\Omega^{3}} d k_{1} d k_{2} d k_{3} \Psi\left(k_{1}, k_{2}, k_{3}\right)\left|k_{1}, k_{2}, k_{3}\right\rangle
$$

where the wave function $\Psi\left(k_{1}, k_{2}, k_{3}\right)$ is symmetric with respect to exchange of any pair of particles. From the stationary Schrödinger equation $H|\Psi\rangle=E|\Psi\rangle$, using the conservation of total quasimomentum $K$, we obtain [21]

$$
\Psi\left(k_{1}, k_{2}, k_{3}\right)=-\frac{M\left(k_{1}\right)+M\left(k_{2}\right)+M\left(k_{3}\right)}{\epsilon\left(k_{1}, k_{2}, k_{3}\right)-E},
$$

where functions $M(k)$ satisfy the 1D Mattis integral equation [21]

$$
M(k)\left[1+I_{E}(k)\right]=-\frac{U}{\pi} \int_{-\pi}^{\pi} d q \frac{M(q)}{\epsilon(k, q, K-k-q)-E},
$$

with $I_{E}(k)$ being a generalized Watson integral [22]

$$
I_{E}(k) \equiv \frac{U}{2 \pi} \int_{-\pi}^{\pi} d q \frac{1}{\epsilon(k, q, K-k-q)-E}=-\frac{\operatorname{sgn}[E-\epsilon(k)] U}{\sqrt{[E-\epsilon(k)]^{2}-16 J^{2} \cos ^{2}[(K-k) / 2]}}
$$

Equation (19) can be cast as a homogeneous Fredholm equation of the second kind with eigenvalue $\lambda=1$. Hence, for a given $U / J$ and fixed $K$, it is a nonlinear equation for energy $E$, which we solve numerically.

The full three-body spectrum of Hamiltonian (1) is shown in Fig. 3left panel, with the bound state energies denoted by $E_{b}, E_{a 1}$ and $E_{a 2}$. For concreteness, here we consider attractive interaction, $U<0$, but note that our results equally apply to the case of repulsive interaction, $U>0$ [15, 20].

In complete analogy with the two-body problem, the Bose-Hubbard Hamiltonian (11) with $|U| / J \gg 1$ has a very narrow band of on-site bound states, corresponding to three tightly bound bosons co-localized on the same lattice site, with energies $E_{b} \approx 3 U$ far from both scattering continua [21]. But as also seen in Fig. 3, in a 1D lattice, bosons can form two new kinds of three-body bound states whose energies $E_{a 1}$ and $E_{a 2}$ lie below and above the two-body continuum $E_{c 2}$. Some properties of these states can be deduced by energy considerations. First, these are not on-site bound states, since their energies $E_{a 1(2)} \simeq U+O(J)$ are far from $3 U$. Next, their binding energies, with respect to the $E_{c 2}$ band, are $E_{B 1(2)} \lesssim \mp J / 2$, which suggests that these are off-site weakly-bound states of a dimer and a monomer. Note that the state above the two-body continuum is bound stronger than the state below the continuum. Finally, they are not Efimov states which can exist only in 3D systems near two-boson resonances [21, 23].

As can be seen from Fig. 3 right panel, where we plot the binding energies $E_{B 1(2)}$ at quasimomentum $K=0$, there are thresholds for the existence of full bands $(K \in[-\pi, \pi])$ of the off-site bound states. For the trimer below the two-body continuum, the binding energy vanishes when $|U| \approx 4 J$ : at this critical value of $U$ the trimer energy $E_{a 1}$ approaches the edge of the dimer-monomer scattering continuum $E_{c 2}=-\sqrt{U^{2}+16 J^{2}}-2 J$. On the other hand, the $K=0$ trimer above the two-body continuum ceases to exist already for $|U| \approx 8.5 J$, since then its energy $E_{a 2}$
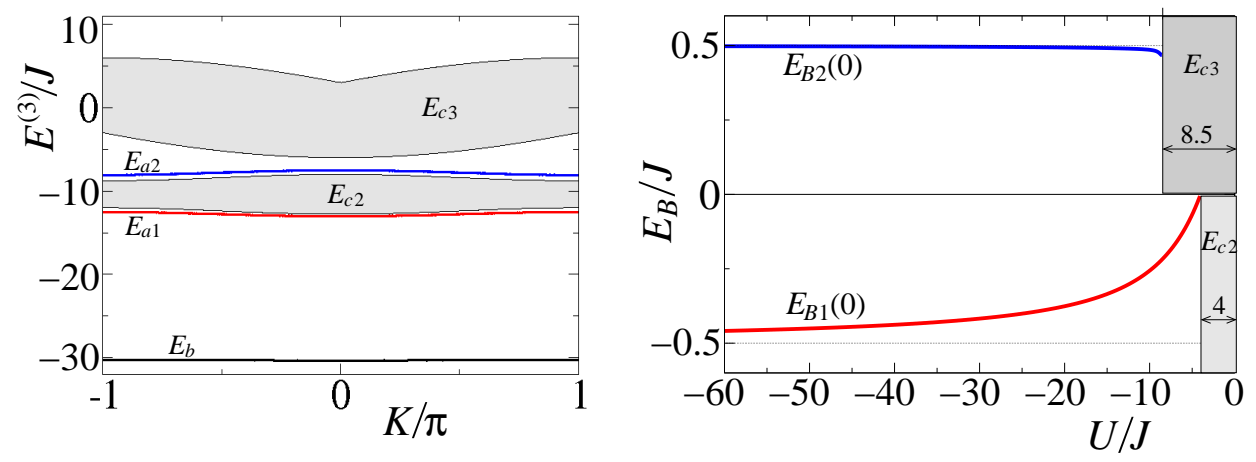

FIG. 3: Left: Full three-particle energy spectrum of Hamiltonian (1) with $U=-10 J$, versus the total quasimomentum $K$. All bound states are obtained via exact numerical solution of Eq. (19). Right: Binding energies $E_{B}$ for the off-site (weakly-bound) trimers at $K=0$ versus the interaction strength $U<0$. 
approaches the bottom of the three-body continuum $E_{c 3}=3 \epsilon(0)=-6 J$ (the two continua, $E_{c 2}$ and $E_{c 3}$, overlap for $|U| \leq 8 J)$. Thus, at $K=0$, the trimer state with energy $E_{a 2}$ starts to appear well in the strong interaction regime, while for larger $K$ the threshold is smaller: $|U| \approx 4 J$ for $|K| \rightarrow \pi$.

\section{Effective model}

Since for strong on-site interaction $|U| / J \gg 1$ the dimer is essentially unbreakable, the off-site trimers with energies close to $U$ must be bound due to a mechanism different from the on-site interaction alone. To identify such a mechanism, we derive an effective perturbative model, valid for $|U| / J>8$, describing two distinguishable, hard-core particles - the dimer and the monomer. To second order in the tunneling rate $J$, the effective Hamiltonian reads

$$
H_{\text {eff }}=H_{1}+H_{2}+H_{\text {int }},
$$

where

$$
H_{1}=-J \sum_{j}\left(\hat{b}_{j}^{\dagger} \hat{b}_{j+1}+\text { H.c. }\right)
$$

describes the single monomer;

$$
H_{2}=\mathcal{E}^{(2)} \sum_{j} \hat{m}_{j}-J^{(2)} \sum_{j}\left(\hat{c}_{j}^{\dagger} \hat{c}_{j+1}+\text { H.c. }\right)
$$

is the Hamiltonian for a dimer (cf. Eq. (16)), with $\hat{c}_{j}^{\dagger}\left(\hat{c}_{j}\right)$ being the dimer creation (annihilation) operator and $\hat{m}_{j}=\hat{c}_{j}^{\dagger} \hat{c}_{j}$ the number operator at site $j$; and finally

$$
H_{\mathrm{int}}=V^{(2)} \sum_{j} \hat{m}_{j} \hat{n}_{j \pm 1}-W \sum_{j}\left(\hat{c}_{j+1}^{\dagger} \hat{c}_{j} \hat{b}_{j}^{\dagger} \hat{b}_{j+1}+\text { H.c. }\right)
$$

describes effective interactions between the dimer and the monomer, including a weak nearest-neighbor interaction $V^{(2)}=-7 J^{2} / 2 U$, and an exchange interaction with the rate $W=2 J$. As we will see below, it is the exchange term that is responsible for the formation of the off-site trimers.

In Fig. 4 right panel, we plot the spectrum of the effective Hamiltonian (20), which contains two bound states with energies $E_{a 1}$ and $E_{a 2}$ below and above the two-body scattering continuum $E_{c 2}=\mathcal{E}^{(2)}+\epsilon^{(2)}(Q)+\epsilon(K-Q)$. These dimer-monomer bound states are obtained using the Schrödinger equation for the two-body wave function $\Psi(Q, k)$ in momentum space, which leads to the integral equation

$$
\Psi(Q, k)=-\frac{1}{2 \pi} \int_{-\pi}^{\pi} d q \frac{U_{12}+V_{\cos }(Q, q)+V_{\sin }(Q, q)}{\mathcal{E}^{(2)}+\epsilon^{(2)}(q)+\epsilon(K-q)-E} \Psi(q, k),
$$

where $K=Q+k$ is the total quasimomentum, $V_{\cos }(Q, q)=\left[2 V^{(2)} \cos (q)-4 J \cos (K-q)\right] \cos (Q)$ and $V_{\sin }(Q, q) \equiv$ $V_{\cos }(Q, q)$ with $\cos \rightarrow \sin$, while $U_{12}(\rightarrow \infty)$ is an artificial dimer-monomer on-site interaction imposing the hardcore condition on Hamiltonian (20). Equation (21) reduces to a non-linear equation for the energy $E$ solving which (numerically) we obtain $E_{a 1}$ and $E_{a 2}$. Comparison with the exact spectrum on the left panel of Fig. 4 reveals good agreement: the continuum spectra are indistinguishable, while the small but noticeable differences in the bound-state energies are associated with the internal structure of the dimer, not accounted for by the effective model, and they gradually disappear with increasing the on-site interaction strength $U$.

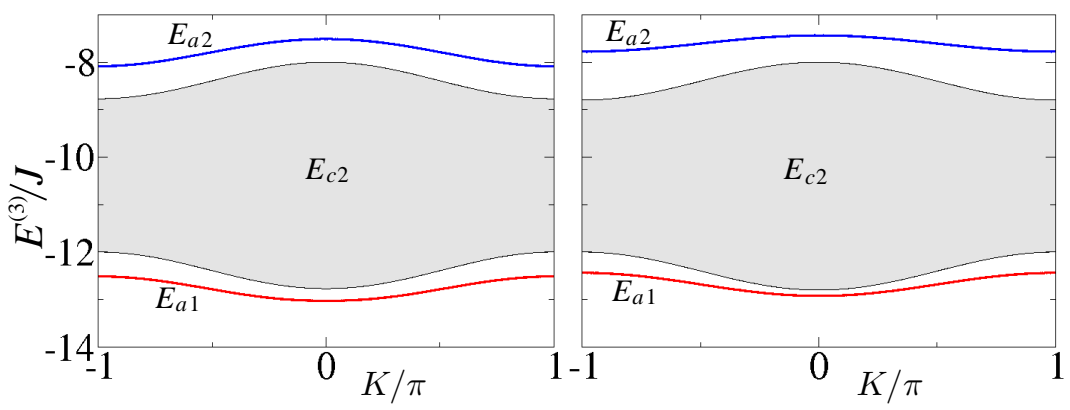

FIG. 4: Left: Magnified part of the exact three-particle spectrum of Fig. 3. Right: Dimer-monomer spectrum of the effective Hamiltonian (20) with the two bound states obtained via numerical solution of Eq. (21). 


\section{Analytic solutions}

There are two important cases, corresponding to the maximal $(K=\pi)$ and minimal $(K=0)$ total quasimomentum $K$, for which the bound and scattering states of the effective Hamiltonian (20) can be calculated analytically employing the method of Sec. [II] To that end, we expand the two-particle eigenstates in coordinate basis $|\Psi\rangle=\sum_{j_{1} \neq j_{2}} \Psi\left(j_{1}, j_{2}\right)\left|j_{1}, j_{2}\right\rangle$ with the wave function in the form $\Psi\left(j_{1}, j_{2}\right)=e^{i K j_{R}} e^{-i \beta_{K} j_{r}} \psi_{K}\left(j_{r}\right)$, where $j_{R} \equiv \frac{1}{2}\left(j_{1}+j_{2}\right), j_{r} \equiv j_{1}-j_{2}$, with $j_{1}$ and $j_{2}$ being the lattice positions of the monomer and dimer, and $\tan \left(\beta_{K}\right)=\tan (K / 2)\left[J-J^{(2)}\right] /\left[J+J^{(2)}\right]$. For the relative coordinate wave function $\psi_{K}\left(j_{r}\right)$, imposing the hardcore condition $\psi_{K}(0)=0$, we then obtain the difference relations

$$
\begin{aligned}
J_{K} \psi_{K}( \pm 2)+W_{K} \psi_{K}(\mp 1)+\left[\bar{E}-V^{(2)}\right] \psi_{K}( \pm 1) & =0 \\
J_{K}\left[\psi_{K}\left(j_{r}+1\right)+\psi_{K}\left(j_{r}-1\right)\right]+\bar{E} \psi_{K}\left(j_{r}\right) & =0
\end{aligned}
$$

with $\left|j_{r}\right|>1, J_{K} \equiv \sqrt{J^{2}+J^{(2) 2}+2 J J^{(2)} \cos (K)}, W_{K} \equiv W \cos (K)$, and $\bar{E} \equiv E-\mathcal{E}^{(2)}$.

a. Scattering solutions: Using the standard ansatz $\psi_{K, k}^{(+)}\left(j_{r}\right)=\cos \left(k\left|j_{r}\right|+\delta_{K, k}^{(+)}\right)$and $\psi_{K, k}^{(-)}\left(j_{r}\right)=$ $\operatorname{sgn}\left(j_{r}\right) \cos \left(k\left|j_{r}\right|+\delta_{K, k}^{(-)}\right)$with $k$ the relative quasimomentum, for the corresponding phase shifts $\delta_{K, k}^{( \pm)}$of the symmetric $(+)$ and antisymmetric $(-)$ scattering wave functions we obtain

$$
\tan \left(\delta_{K, k}^{( \pm)}\right)=\frac{J_{K} \cos (2 k)+\left[\bar{E} \pm W_{K}-V^{(2)}\right] \cos (k)}{J_{K} \sin (2 k)+\left[\bar{E} \pm W_{K}-V^{(2)}\right] \sin (k)},
$$

with $\bar{E}=E_{c 2}-\mathcal{E}^{(2)}=-2 J_{K} \cos (k)$. Note that in the limit of $|U| \rightarrow \infty$, as the the nearest neighbour interaction $V^{(2)}$ and the dimer hopping $J^{(2)}$ tend to zero $\left(J_{K} \rightarrow J\right)$, Eq. (23) holds for all $K=k$. The full scattering wave function is given by a superposition $\psi_{K, k}\left(j_{r}\right)=A \psi_{K, k}^{(-)}\left(j_{r}\right)+B \psi_{K, k}^{(+)}\left(j_{r}\right)$, which, upon expressing through incident, reflected and transmitted waves,

$$
\psi_{K, k}\left(j_{r}\right)= \begin{cases}e^{i k j_{r}}+r_{K} e^{-i k j_{r}} & \left(j_{r}<0\right) \\ t_{K} e^{i k j_{r}} & \left(j_{r}>0\right)\end{cases}
$$

leads to $A / B=-e^{-i\left[\delta_{K, k}^{(+)}-\delta_{K, k}^{(-)}\right]}$. For the reflection $r_{K}(k)$ and transmission $t_{K}(k)$ amplitudes we then obtain $r_{K}, t_{K}=$ $\frac{1}{2}\left[e^{2 i \delta_{K, k}^{(+)}} \pm e^{2 i \delta_{K, k}^{(-)}}\right]$, and the transmission and reflection probabilities are given by $T_{K}=\left|t_{K}\right|^{2}=\sin ^{2}\left(\delta_{K, k}^{(+)}-\delta_{K, k}^{(-)}\right)$ and $R_{K}=\left|r_{K}\right|^{2}=\cos ^{2}\left(\delta_{K, k}^{(+)}-\delta_{K, k}^{(-)}\right)$.

In Fig. 5 we plot $T_{K}(k)$ for $U / J=-10$ at total quasimomenta $K=0$ and $|K|=\pi$. The transmission spectra for the intermediate values of $K$ lies in between the curves for $K=0$ and $K=\pi$. We observe the maximum transmission in the vicinity of $k= \pm \pi / 2$, where $T_{K}(\pi / 2)$ ranges from $50 \%$ to $80 \%$. With increasing the interaction strength $U$, we find that the maximum transmission saturates at around $64 \%$ for all values of $K$, which should be contrasted with the results of Ref. [19].

b. Bound solutions: The dimer-monomer bound states are obtained from Eq. (22) using the exponential ansatz $\psi_{K}\left(j_{r}>0\right) \propto \alpha_{K}^{j_{r}-1}$ and $\psi_{K}\left(-j_{r}\right)= \pm \psi_{K}\left(j_{r}\right)$, which yields

$$
\alpha_{K}^{( \pm)}=-\frac{J_{K}}{V^{(2)} \mp W_{K}}
$$

for the symmetric $(+)$ and antisymmetric $(-)$ wave function of the bound state $\left(\left|\alpha_{K}\right|<1\right)$, with the corresponding energy $\bar{E}_{a 1(2)}=-J_{K}\left[1+\left(\alpha_{K}^{( \pm)}\right)^{2}\right] / \alpha_{K}^{( \pm)}$.

It is now easy to see that without the exchange interaction there would be no dimer-monomer bound states (for any $K)$. Indeed, this hypothetic $(W=0)$ problem is exactly solvable for all $K$, and for two hard-core bosons with nearest-neighbour interaction $V^{(2)}$ there could be only one bound state[14] when $\left|\alpha_{K}\right|=\left|J_{K} / V^{(2)}\right|<1$, which cannot be satisfied in the range of validity $(|U| / J>8)$ of the effective model, Eq. (20). The effective nearest-neighbour interaction is, however, responsible for the asymmetry in the binding energies $E_{B 1(2)}=\bar{E}_{a 1(2)} \mp 2 J_{K}$ of the exchangebound trimers below and above the continuum $E_{c 2}$ (see Fig. 3 right panel). With increasing the on-site interaction $U$, the binding energies attain the universal limits $E_{B 1(2)} \rightarrow \mp J / 2$ which remain valid for all $K$, since $J^{(2)} \rightarrow 0$ as $|U / J| \rightarrow \infty$. We thus conclude that the effective particle exchange interaction binds the dimer and the monomer into the weakly bound trimer. 


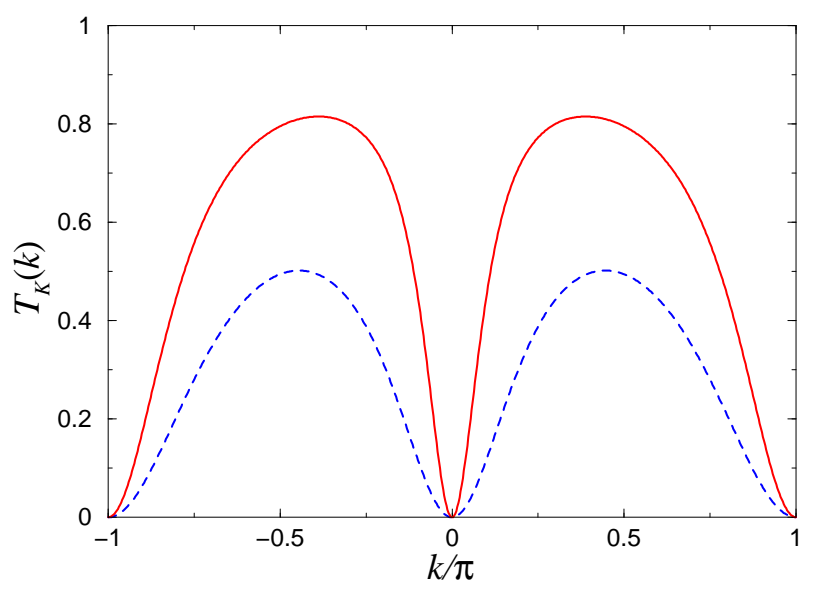

FIG. 5: Transmission probability $T_{K}(k)$ of a single particle with relative quasimomentum $k$ through a bound dimer, for $U / J=-10$ and total quasimomenta $K=0$ (red solid line) and $|K|=\pi$ (blue dashed line).

\section{CONCLUSIONS}

In this paper, we have presented complete solutions for the one-, two- and three-body problems in a 1D tight-binding lattice described by the Bose-Hubbard model.

For the case of two bosons, we have derived the scattering states and the bound states of co-localized particles, which we termed as dimers. The corresponding binding mechanism is provided by the on-site interaction between the particles. Perhaps counterintuitively, these bound dimer states exist not only for attractive, but also for repulsive interactions [8]. In free space, the repulsive interaction would inevitably lead to the pair separation, or dissociation, whereby the potential energy of the repulsion is converted into the kinetic energy of the free particles. In the lattice, however, the kinetic energy of each particle cannot take on arbitrary values, but is restricted to the values in the allowed Bloch band, which itself is bounded both from below and from above. Therefore, in the absence of energy dissipation, a pair of co-localized particles interacting even via repulsive potential is destined to stay bound together as a dimer, simply because there are no available free-particle energy states to which the dimer can dissociate.

For three bosons, we have found three families of trimers - bound states of three particles. The first strongly-bound trimer, being an analog of the dimer, corresponds to all three particles occupying the same lattice site and bound by the on-site interaction. The other two families of trimers are weakly-bound with energies just below and above the two-body scattering continuum of a single particle (monomer) and an interaction-bound dimer. Intuitively, these trimer states correspond to symmetric and antisymmetric states of a dimer and monomer at the neighboring lattice sites interacting with each other via an effective (particle) exchange interaction, which is responsible for their binding.

The phenomena discussed above are pertinent to the experiments with cold bosonic atoms in optical lattices [7]. Studying larger number of bosons in a lattice might reveal other exotic bound states, while longer range interactions, such as those between dipolar atoms [24], or molecules [25], will certainly play an important role in the formation of few-body bound states [14, 15, 26].

\section{Acknowledgements}

This work was supported by the EC Marie-Curie Research Training Network EMALI.

[1] N.J. Ashcroft and and N.D. Mermin, Solid State Physics (Thomson, New York, 1976).

[2] S. Sadchev, Quantum Phase Transitions (Cambridge University Press, Cambridge, 1999).

[3] J. Hubbard, Proc. Roy. Soc. A 276238 (1963).

[4] M.P.A. Fisher, P.B. Weichman, G. Grinstein, and D.S. Fisher, Phys. Rev. B 40, 546 (1989).

[5] D. Jaksch, C. Bruder, J.I. Cirac, C.W. Gardiner, and P. Zoller, Phys. Rev. Lett. 81, 3108 (1998).

[6] M. Greiner, O. Mandel, T. Esslinger, T.W. Hänsch, and I. Bloch, Nature 415, 39 (2002).

[7] I. Bloch, J. Dalibard, and W. Zwerger, Rev. Mod. Phys. 80, 885 (2008). 
[8] K. Winkler, G. Thalhammer, F. Lang, R. Grimm, J. Hecker Denschlag, A.J. Daley, A. Kantian, H.P. Büchler, and P. Zoller, Nature 441, 853 (2006).

[9] R. Piil and K. Mølmer, Phys. Rev. A 76, 023607 (2007).

[10] N. Nygaard, R. T. Piil,and K. Mølmer, Phys. Rev. A 78, 023617 (2008).

[11] R. T. Piil, N. Nygaard and K. Mølmer, Phys. Rev. A 78, 033611 (2008).

[12] M. Valiente and D. Petrosyan, J. Phys. B 41, 161002 (2008).

[13] M. Valiente and D. Petrosyan, Europhys. Lett. 83, 30007 (2008).

[14] M. Valiente and D. Petrosyan, J. Phys. B 42, 121001 (2009).

[15] M. Valiente, arXiv:1001.3805 (2010).

[16] D. Petrosyan, B. Schmidt, J.R. Anglin, and M. Fleischhauer, Phys. Rev. A 76, 033606 (2007).

[17] B. Schmidt, M. Bortz, S. Eggert, M. Fleischhauer and D. Petrosyan, Phys. Rev. A 79, 063634 (2009).

[18] L. Wang, Y. Hao and S. Chen , Eur. Phys. J. D 48, 229 (2008).

[19] L. Jin, B. Chen, and Z. Song, Phys. Rev. A 79, 032108 (2009).

[20] M. Valiente, D. Petrosyan and A. Saenz, Phys. Rev. A 81, 011601(R) (2010).

[21] D.C. Mattis, Rev. Mod. Phys. 58, 361 (1986).

[22] G.N. Watson, Q. J. Math. Oxford 10, 266 (1939).

[23] V.N. Efimov, Sov. J. Nucl. Phys. 12, 589 (1971).

[24] A. Griesmaier, J. Werner, S. Hensler, J. Stuhler, and T. Pfau, Phys. Rev. Lett. 94, 160401 (2005).

[25] K.-K. Ni, S. Ospelkaus, M.H.G. de Miranda, A. Pe'er, B. Neyenhuis, J.J. Zirbel, S. Kotochigova, P.S. Julienne, D.S. Jin, and J. Ye, Science 322, 231 (2008).

[26] M. Valiente, Ph.D. Thesis (unpublished).

[27] Contrast, however, with the extended Hubbard model in Refs. 14, 15] 